\title{
Effect of gravitational acceleration on unsteady biomagnetic fluid flow
}

\author{
Nor Amirah Idris*, Norsarahaida Amin, Hamisan Rahmat \\ Department of Mathematical Sciences, Faculty of Sciences, Universiti Teknologi Malaysia, Johor Bahru, Malaysia
}

\section{Email address:}

mira9446@gmail.com (N. A. Idris), norsarahaida@utm.my (N. Amin), hamisan@utm.my (H. Rahmat)

\section{To cite this article:}

Nor Amirah Idris, Norsarahaida Amin, Hamisan Rahmat. Effect of Gravitational Acceleration on Unsteady Biomagnetic Fluid Flow. Applied and Computational Mathematics. Vol. 3, No. 6, 2014, pp. 285-294. doi: 10.11648/j.acm.20140306.11

\begin{abstract}
This paper investigates the effect of gravitational acceleration on unsteady biomagnetic fluid flow in a channel under the influence of a spatially varying magnetic field. The study on biomagnetic fluid under the action of an applied magnetic field is important in the development of Biomagnetic Fluid Dynamics (BFD). Most existing studies analyze flows in steady state conditions and the effect of gravitational acceleration has not been addressed. For the mathematical model, the Navier-Stokes equations, energy equation and an additional term that describes the magnetic force and gravitational effect which is consistent with the principles of ferrohydrodynamics (FHD) are employed. The nonlinear governing differential equations are non-dimensionalized and then discretized based on a finite difference technique on a staggered grid system. The solution of these problems is obtained numerically using pressure correction method with SIMPLE algorithm. For a range of governing parameters such as the magnetic number $M n_{F}$ and Richardson number $R i$, the numerical results show that the gravitational acceleration has a profound effect on both velocity and temperature profiles. The streamlines plotted also show that vortices appear near the lower plate where the magnetic source is located.
\end{abstract}

Keywords: Gravitational Acceleration, Biomagnetic Fluid Flow, Unsteady, FHD, SIMPLE Algorithm

\section{Introduction}

Biomagnetic Fluid Dynamics (BFD) is the study of the flow of biological fluid in the presence of magnetic field. As fluid moves about in a living creature, its flow is influenced by the presence of the magnetic field. An example of biomagnetic fluid is blood. Tzirtzilakis and Kafoussias [16] stated magnetic field is created in blood by the interaction of cell membrane, intercellular protein, and hemoglobin molecule which is a form of iron oxide existing at a uniquely high concentration in mature red blood cells.

The magnetization property $M$ is the behaviour of a biological fluid when it is exposed to magnetic field. This measures how much the magnetic field is affecting the fluid in various aspects. There are various equations describing the dependence of $M[2,3,6,16,17]$. Mathematical models have been developed to investigate BFD. According to the model of Haik et al. [7] biological fluids are treated as Newtonian, isothermal and consistent with the principles of ferrohydrodynamics (FHD). FHD is the study of electrically non-conducting magnetic fluids. The mathematical formulation based on the principle of FHD was proposed by
Tzirtzilakis et al. [18]. The flow is three-dimensional and isothermal.

In recent years, studies have been made to analyse the effects of magnetic fields and temperature variation in biological fluid. An application of this is in using heat in fluid in eye therapy. Fertman [5] reported on how magnetic fluid was injected to induce hyperthemia in eye treatment. The treatment of a group of patients was made possible without using anti-inflammatory medication by using this method [5]. In a study on wound treatment, it was reported that the temperature increased more than $3^{\circ} \mathrm{C}$ after magnetic field was applied to a wounded area and the wounds closed after $21-26$ days [12]. On similar wounds which were not treated magnetically, scabs and ulcers were formed even after 50 days. Tzirtzilakis [14] and Tzirtzilakis et al. [15] extended the BFD mathematical model of Haik et al. [7]. Their BFD models are different from [7] in that they dealt with temperature distribution for non-isothermal fluid. Loukopoulos et al. [8] and Papadopoulos et al. [11] derived FHD for steady, two-dimensional and non-isothermal fluid flow. They found a vortex is formed at the lower plate where the magnetic source was located. The temperature, skin friction and rate of heat 
transfer were increasing at the area of magnetic source. Tzirtzilakis and Kafoussias [16] presented the flow of heated ferrofluid over a linearly stretching sheet in the presence of an applied magnetic field due to a magnetic dipole.

The study of BFD is influenced by the principles of FHD and Magnetohydrodynamics (MHD). MHD deals with conducting fluids which ignores the effect of polarization and magnetization. The mathematical model for blood flow in magnetic field consistent with the principles of FHD and MHD in a straight rectangular duct was proposed by Tzirtzilakis [19]. The applications of the applied magnetic field are related to two cases which are a spatially varying magnetic field and a constant magnetic field. Tzirtzilakis and Loukopoulos [20] studied the laminar biomagnetic fluid flow problem while Tzirtzilakis et al. [21] dealt with the turbulent biomagnetic fluid flow in a channel under the influence of a steady localized magnetic field. When the biofluid enters and leaves the locally applied magnetic field, where the gradient of the magnetic field strength is high, the magnetization force and Lorentz force appear. Therefore, the BFD model for [20, 21] considered FHD and MHD principles. Two vortices appeared at the area of two points where the magnetic field started and stopped to apply. In the case of turbulent flow, the skin friction and heat transfer were greater compared to the laminar flow. The problem of biomagnetic fluid flow under the influence of a spatially varying magnetic field was developed by Nursalasawati [10]. She assumed the magnetisation force was due to FHD interaction. From her investigation, the results regarding the velocity showed that the presence of magnetic field appreciably influenced the flow field. In addition, she considered the Lorentz and magnetisation forces due to MHD and FHD interaction. The results are similar to the case of FHD interaction only. The Lorentz force just gives a small influence to the flow behaviours.

Saha et al. [13] investigated various ranges of Richardson number for combined free and forced convection inside a two-dimensional multiple ventilated rectangular enclosure. The unsteady flow past a uniformly accelerated infinite vertical plate in the presence of variable temperature and mass flux was proposed by Muthucumaraswamy et al. [9]. The results indicated that the velocity increased with increasing values of thermal Grashof number or mass Grashof number.

Various methodologies for numerical solutions are adopted in order to solve the biomagnetic fluid flow problems. The stream function-vorticity formulation had been used for the numerical investigation of the steady biomagnetic fluid flow in a channel under a spatially varying magnetic field $[8,14$, 15]. Tzirtzilakis [19] adopted a pressure-linked pseudotransient method for solving biomagnetic fluid flow in a straight rectangular duct under the action of a uniform or a spatially varying magnetic field. In that study, a finite difference numerical technique was used for discretization.
SIMPLE method was used for the problem of biomagnetic fluid flow in a curved square duct under the influence of an applied magnetic field [11].

In the present study, the effect of gravitational acceleration on biomagnetic fluid flow in a channel is investigated. The flow is considered to be unsteady, two-dimensional, non-isothermal, laminar and incompressible. The magnetization equation is assumed by a linear equation involving the magnetic field intensity $H^{*}$ and temperature $T^{*}$. This model is solved using a finite difference numerical technique based on Semi-Implicit Method for Pressure Linked Equations (SIMPLE) algorithm on a staggered grid system. The result concerning the velocities and temperature fields are observed and explained.

\section{Mathematical Model}

We consider the unsteady, two-dimensional, laminar and viscous fluid flow between two parallel flat plates. The distance between the plates is $h^{*}$ and the length of the plates is $L^{*}$. The fluid is assumed to have Newtonian behavior, is homogeneous and electrically non-conducting and is governed by FHD principle.

In BFD problem, a spatially varying magnetic field is taken into account. According to Nursalasawati [10], the existence of the Lorentz force gaves only a small influence to the flow behaviours, but the energy equation played an important role in some observation as stated by Loukopoulos and Tzirtzilakis [8]. Erwan [4] stated that the Lorentz force governed by MHD principle did not affect the flow field. Thus, for the present study, we ignore the MHD but include the energy equation in modeling the flow behaviour.

In this case, the gravitational acceleration and energy equation are considered. The upper plate temperature is kept at a constant $T_{u}^{*}$, while the lower at $T_{l}^{*}$, such that $T_{u}^{*}>T_{l}^{*}$. The governing equations of the unsteady Navier-Stokes and energy equations under the influence of the spatially varying magnetic field and gravitational acceleration are given below:

$$
\begin{gathered}
\frac{\partial u^{*}}{\partial x^{*}}+\frac{\partial v^{*}}{\partial y^{*}}=0 \\
\frac{\partial u^{*}}{\partial t^{*}}+u^{*} \frac{\partial u^{*}}{\partial x^{*}}+v^{*} \frac{\partial u^{*}}{\partial y^{*}}=-\frac{1}{\rho^{*}} \frac{\partial p^{*}}{\partial x^{*}}+v^{*}\left(\frac{\partial^{2} u^{*}}{\partial x^{* 2}}+\frac{\partial^{2} u^{*}}{\partial y^{* 2}}\right)+ \\
\frac{\mu_{0}{ }^{*} M^{*}}{\rho^{*}} \frac{\partial H^{*}}{\partial x^{*}} \\
\frac{\partial u^{*}}{\partial t^{*}}+u^{*} \frac{\partial u^{*}}{\partial x^{*}}+v^{*} \frac{\partial u^{*}}{\partial y^{*}}=-\frac{1}{\rho^{*}} \frac{\partial p^{*}}{\partial x^{*}}+v^{*}\left(\frac{\partial^{2} u^{*}}{\partial x^{* 2}}+\frac{\partial^{2} u^{*}}{\partial y^{* 2}}\right)+ \\
\frac{\mu_{o}^{*} M^{*}}{\rho^{*}} \frac{\partial H^{*}}{\partial y^{*}}+g^{*} \beta^{*}\left(T-T_{c}\right)
\end{gathered}
$$




$$
\begin{gathered}
\rho^{*} c_{p}^{*}\left(\frac{\partial T^{*}}{\partial t^{*}}+u^{*} \frac{\partial T^{*}}{\partial x^{*}}+v^{*} \frac{\partial T^{*}}{\partial y^{*}}\right)=k^{*}\left(\frac{\partial^{2} T^{*}}{\partial x^{* 2}}+\frac{\partial^{2} T^{*}}{\partial y^{* 2}}\right)+\mu_{0}^{*} T^{*} \frac{\partial M^{*}}{\partial T^{*}}\left(u^{*} \frac{\partial H^{*}}{\partial x^{*}}+v^{*} \frac{\partial H^{*}}{\partial y^{*}}\right)+ \\
\mu^{*}\left[2\left(\frac{\partial u^{*}}{\partial x^{*}}\right)^{2}+2\left(\frac{\partial v^{*}}{\partial y^{*}}\right)^{2}+\left(\frac{\partial v^{*}}{\partial x^{*}}+\frac{\partial u^{*}}{\partial y^{*}}\right)^{2}\right]
\end{gathered}
$$

The boundary conditions are

$$
\begin{aligned}
& x^{*}=0,0 \leq y^{*} \leq h^{*}: u^{*}=u_{r}^{*}, v^{*}=0, T^{*}=T^{*}\left(y^{*}\right), \\
& x^{*}=L^{*}, 0 \leq y^{*} \leq h^{*}: \partial u^{*} / \partial x^{*}=0, \partial v^{*} / \partial x^{*}=0, \partial v^{*} / \partial x^{*}=0, \\
& y^{*}=h^{*}, 0 \leq x^{*} \leq L^{*}: u^{*}=0, v^{*}=0, T^{*}=T_{u}^{*}, \\
& y^{*}=0,0 \leq x^{*} \leq L^{*}: \quad u^{*}=0, v^{*}=0, T^{*}=T_{l}^{*} .
\end{aligned}
$$

In the above equations, the asterisk superscripts for the quantities denote that they are dimensional. $u^{*}$ and $v^{*}$ are the fluid velocity components in the $x$ and $y$ directions, $p^{*}$ is the pressure, $\rho^{*}$ the constant density of the fluid, $v^{*}=\mu^{*} / \rho^{*}$ the kinematic viscosity, $\mu_{o}^{*}$ the magnetic permeability in vacuum, $M^{*}$ the magnetization, $H^{*}$ the magnetic field intensity, $g^{*}$ the acceleration due to gravity and $k^{*}$ the thermal conductivity and $T^{*}$ the temperature where $T_{u}^{*}$ and $T_{l}^{*}$ are constants. The terms $\mu_{0}{ }^{*} M^{*} \partial H^{*} / \rho^{*} \partial x^{*}$ and $\mu_{o}^{*} M^{*} \partial H^{*} / \rho^{*} \partial y^{*}$ in (2) and (3) are the components of the magnetic force per unit volume and dependent on the magnetic gradient. These terms are derived based on FHD principle.

The term $g^{*} \beta^{*}\left(T-T_{c}\right)$ in (3) represents the density which varies linearly with temperature. Temperature difference will cause the density difference. This situation is characterized by the volumetric thermal expansion coefficient, $\beta^{*}$.The derivation of this coefficient is shown below:

$$
\begin{gathered}
\beta^{*}=-\frac{1}{\rho}\left(\frac{\partial \rho}{\partial T}\right) p \approx-\frac{1}{\rho} \frac{\rho_{\infty}-\rho}{T_{\infty}-T}=-\frac{1}{\rho} \frac{\Delta \rho}{\Delta T} \\
\Delta \rho \approx \beta^{*} \Delta T
\end{gathered}
$$

The equation of magnetization with the magnetic field intensity $H^{*}$ and temperature $T^{*}$ is assumed to be [14]:

$$
M^{*}=K^{*} H^{*}\left(T_{c}^{*}-T\right)
$$

where $K^{*}$ is a constant and $T_{c}^{*}$ is the Curie temperature. The magnetic field intensity is given as [14]:

$$
\begin{aligned}
H^{*}\left(x^{*}, y^{*}\right) & =\left[H_{x}^{* 2}+H_{y}^{* 2}\right]^{1 / 2} \\
& =\frac{\gamma}{2 \pi} \frac{1}{\sqrt{\left(x^{*}-a\right)^{2}+\left(y^{*}-b\right)^{2}}}
\end{aligned}
$$

where $(a, b)$ is the position of the magnetic source and $\gamma$ is the magnetic field strength at the source.

\subsection{Non-Dimensionalization}

In order to proceed to numerical solution of the system (1) to (4) with boundary conditions and assumptions (5) and (6), the following non-dimensional variables are chosen:

$$
\left.\begin{array}{l}
t=\frac{t^{*} u_{r}^{*}}{h^{*}}, x=\frac{x^{*}}{h^{*}}, y=\frac{y^{*}}{h^{*}}, u=\frac{u^{*}}{u_{r}{ }^{*}}, v=\frac{v^{*}}{u_{r}{ }^{*}}, \\
p=\frac{p^{*}}{\rho u_{r}{ }^{* 2}}, H=\frac{H^{*}}{H_{r}{ }^{*}}, T=\frac{T^{*}-T_{l}^{*}}{T_{u}{ }^{*}-T_{l}{ }^{*}} .
\end{array}\right\}
$$

where $u_{r}^{*}$ is the maximum velocity at the entrance and $H_{r}^{*}$ is the magnetic field strength. After using the dimensionless definitions as (7) the set of equations above will become as follows.

$$
\begin{gathered}
\frac{\partial u}{\partial x}+\frac{\partial v}{\partial y}=0 \\
\frac{\partial u}{\partial t}+\frac{\partial u}{\partial x}+v \frac{\partial u}{\partial y}=\frac{\partial p}{\partial x}+\frac{1}{R e}\left(\frac{\partial^{2} u}{\partial x^{2}}+\frac{\partial^{2} u}{\partial y^{2}}\right)+M n_{F} H \frac{\partial H}{\partial x} \\
\frac{\partial v}{\partial t}+\frac{\partial v}{\partial x}+v \frac{\partial v}{\partial y}=-\frac{\partial p}{\partial y}+\frac{1}{R e}\left(\frac{\partial^{2} v}{\partial x^{2}}+\frac{\partial^{2} v}{\partial y^{2}}\right)+ \\
\frac{\partial T}{\partial t}+u \frac{\partial T}{\partial x}+v \frac{\partial T}{\partial y}=\frac{1}{P e}\left(\frac{\partial^{2} T}{\partial x^{2}}+\frac{\partial^{2} T}{\partial y^{2}}\right)^{+} \frac{\partial H}{\partial y}+R i T \\
M n_{F} E c\left(u H \frac{\partial H}{\partial x}+v H \frac{\partial H}{\partial y}\right)(\varepsilon-T)+ \\
\frac{E c}{R e}\left[2\left(\frac{\partial u}{\partial x}\right)^{2}+2\left(\frac{\partial v}{\partial y}\right)^{2}+\left(\frac{\partial v}{\partial x}+\frac{\partial u}{\partial y}\right)^{2}\right]
\end{gathered}
$$

where $R e=h^{*} \rho^{*} u_{r}^{*} / \mu^{*}$ is the Reynolds number, $M n_{F}=\mu_{0}^{*} M_{0}^{*} / \rho^{*} u_{r}^{* 2}$ the magnetic number, $\operatorname{Pr}=c_{p}^{*} \mu^{*} / k^{*}$ the Prandtl number, $P e=\operatorname{Re} P r$ the Peclet number $E c=u_{r}^{* 2} / c_{p}^{*}\left(T_{u}^{*}-T_{l}^{*}\right)$ the Eckert number, $\varepsilon=T_{u}^{*} / T_{u}^{*}-T_{l}^{*}$ the temperature number and $G r=g \beta^{*}\left(T_{u}^{*}-T_{l}^{*}\right) h^{3} / v^{2}$ the dimensionless parameter of Grashof number which approximates the ratio of the buoyancy force to viscous force that acting on a fluid. $R i=G r / R e^{2}$ the Richardson number.

For the biomagnetic fluid flow problem, the dimensionless parameter appearing in (9) to (11) which is the magnetic 
number is developed as:

$$
\begin{aligned}
M n_{F} & =\frac{\mu_{0}^{*} H_{r}^{* 2} K^{*}\left(T_{u}^{*}-T_{l}^{*}\right)}{\rho^{*} u_{r}^{* 2}}=\frac{\mu_{0}^{*} H_{r}^{*} K^{*} H_{r}^{*}\left(T_{u}^{*}-T_{l}^{*}\right)}{\rho^{*} u_{r}^{* 2}} \\
& =\frac{B_{r}^{*} M_{r}^{*}}{\rho^{*} u_{r}^{* 2}}=\frac{B_{r}^{*} M_{r}^{*} h^{* 2} \rho^{*}}{\mu^{* 2} R e^{2}}
\end{aligned}
$$

where $B_{r}^{*}=\mu_{o}^{*} H_{r}^{*}$ the magnetic induction and $M_{r}^{*}$ the magnetization at point $(a, 0)$. We note that, from the definition of the Reynolds number, $u_{r}^{*}=\mu^{*} R e / h^{*} \rho^{*}$. By using the relations (6) and (7), the magnitude of the magnetic field intensity $H$ is as follows [23].

$$
H(x, y)=\frac{|b|}{\sqrt{(x-a)^{2}+(y-b)^{2}}}
$$

The boundary conditions are

$$
\left.\begin{array}{l}
x=0,0 \leq y \leq 1: u=1, \quad v=0, \partial T / \partial x=0, \\
x=1,0 \leq y \leq 1: \partial u / \partial x=0, \partial v / \partial x=0, \partial T / \partial x=0, \\
x=0 \text { or } x=1 \text { and } 0 \leq y \leq 1: u=v=0, T=1, \\
y=0 \text { or } y=1 \text { and } 0 \leq x \leq 1: u=v=0, T=0 .
\end{array}\right\}
$$

\section{Numerical Procedure}

The solution of the governing equations presented in the previous section equations (8) to (11) are discretized by using finite difference based on the staggered grids system. The $u$ momentum equation is approximated by using forward difference around the points $(i+1 / 2, j)$, while the $v$ momentum equation uses forward differences at the points $(i, j+1 / 2)$. For the continuity and energy equations, we will applying central differences at the points $(i, j)$.

There are some important advantages when we stagger the grids. First, staggered grids prevent odd-even coupling or what is known as checkerboarding between the pressure field and the velocity field. Besides that, the continuity equation can be written at the node $(i, j)$ with second order accurate central differences without interpolation of the relevant velocity components. No interpolation is required when we use staggered grid because the variables are stored where they are needed.

\subsection{Discretization of Momentum and Continuity Equations}

From (8) to (11), there are four equations for the four dependent variables $u, v, p$ and $T$. For the energy equation, the temperature gradient exists in the flow. After the velocities and pressure fields are obtained, the temperature field can be solved directly from the energy equation.

Using finite differences, we discretize the variables of the continuity and momentum equations. In this study also, we choose to use the Semi-Implicit Method for Pressure Linked Equations (SIMPLE). We begin with the discretization of (8) to (11) using a first order forward differences in time and central differences for the spatial derivative.

First, for $u$ - momentum in (9), by using central difference around $(i+1 / 2, j)$ the equation becomes

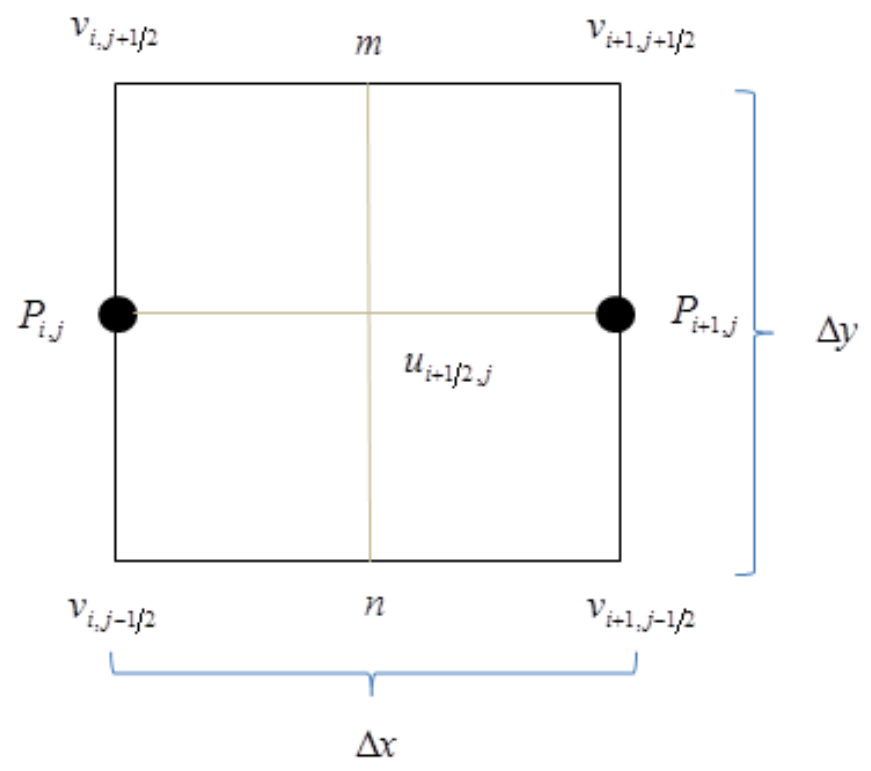

Figure 1. Computational module for $u$-momentum equation

$$
\begin{gathered}
(u)_{i+1 / 2, j}^{n+1}=(u)_{i+1 / 2, j}^{n}+\Delta t A-\frac{\Delta t}{\Delta x}\left(p_{i+1 . j}^{n}-p_{i . j}^{n}\right)+ \\
M n_{F} H \frac{\partial H}{\partial x}
\end{gathered}
$$

where

$$
\begin{aligned}
A= & -\left[\frac{\left(u^{2}\right)_{i+3 / 2, j}^{n}-\left(u^{2}\right)_{i-1 / 2, j}^{n}}{2 \Delta x}+\frac{(u \bar{v})_{i+1 / 2, j+1}^{n}-(u v)_{i+1 / 2, j-1}^{n}}{2 \Delta y}\right]+ \\
& \frac{1}{\operatorname{Re}}\left[\frac{u_{i+3 / 2, j}^{n}-2 u_{i+1 / 2, j}^{n}+u_{i-1 / 2, j}^{n}}{(\Delta x)^{2}}+\frac{u_{i+1 / 2, j+1}^{n}-2 u_{i+1 / 2, j}^{n}+u_{i+1 / 2, j-1}^{n}}{(\Delta y)^{2}}\right]
\end{aligned}
$$

From the discretization of (15), we will need average value of $v$ on the top and bottom at the points $m$ and $n$. The linear interpolation between two adjacent points is defined as follows:

Average values of $v$ at the points $m$ and $n$

$$
\begin{aligned}
& \text { At point } m: \bar{v}_{j+1 / 2}=1 / 2\left(v_{i, j+1 / 2}+v_{i+1, j+1 / 2}\right) \\
& \text { At point } n: v_{j-1 / 2}=1 / 2\left(v_{i, j-1 / 2}+v_{i+1, j-1 / 2}\right)
\end{aligned}
$$

We substitute (16) and (17) into (15).

Next, for $v$ - momentum, we choose to use forward difference centered on point $(i, j+1 / 2)$ for (10) as shown in Fig. 2 below. 


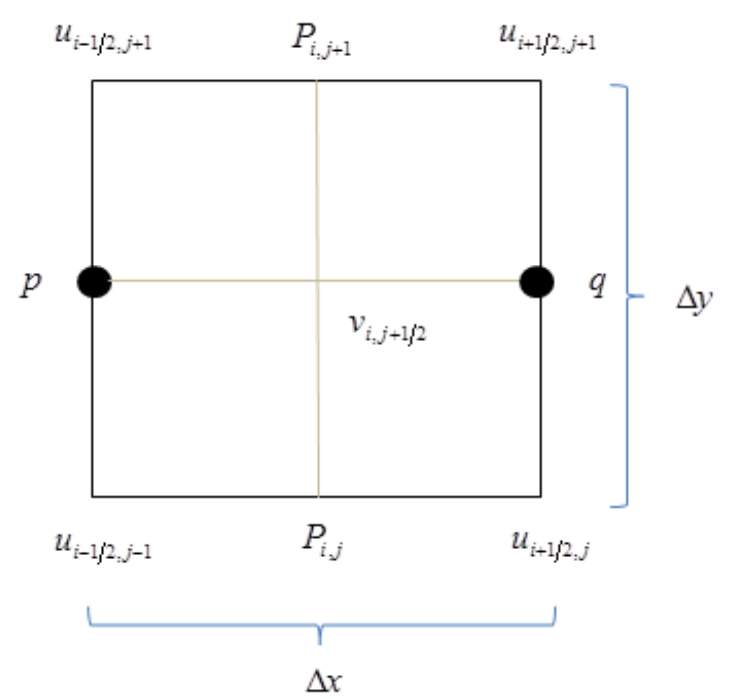

Figure 2. Computational module for $v$-momentum equation

$$
\begin{gathered}
(v)_{i, j+1 / 2}^{n+1}=(v)_{i, j+1 / 2}^{n}+\Delta t B-\frac{\Delta t}{\Delta y}\left(p_{i, j+1}^{n}-p_{i . j}^{n}\right)+ \\
M n_{F} H \frac{\partial H}{\partial y}+R i T_{i, j}
\end{gathered}
$$

where

$$
\begin{aligned}
B & =-\left[\frac{(v \bar{u})_{i+1, j+1 / 2}^{n}-(v u)_{i-1, j+1 / 2}^{n}}{2 \Delta x}+\frac{\left(v^{2}\right)_{i, j+3 / 2}^{n}-\left(v^{2}\right)_{i, j-1 / 2}^{n}}{2 \Delta y}\right]+ \\
& \frac{1}{\operatorname{Re}}\left[\frac{v_{i+1, j+1 / 2}^{n}-2 v_{i, j+1 / 2}^{n}+v_{i-1, j+1 / 2}^{n}}{(\Delta x)^{2}}+\frac{v_{i, j+3 / 2}^{n}-2 v_{i, j+1 / 2}^{n}+v_{i, j-1 / 2}^{n}}{(\Delta y)^{2}}\right]
\end{aligned}
$$

At the points $p$ and $q$ on the left and right side of the edge of the control volume as shown in Figure 2, we define the average values of $u$ as follows:

Average values of $u$ at the point $p$ and $q$

$$
\begin{array}{lll}
\text { At point } p & : & \bar{u}_{i+1 / 2}=1 / 2\left(u_{i+1 / 2, j}+u_{i+1 / 2, j+1}\right) \\
\text { At point } q & : & u_{i-1 / 2}=1 / 2\left(u_{i-1 / 2, j}+v_{i-1 / 2, j+1}\right)
\end{array}
$$

We substitute (19) and (20) into (18).

For continuity equation, applying central difference at point $(i, j)$, we get

$$
a p_{i . j}^{\prime n}+b p_{i+1 . j}^{\prime n}+b p_{i-1 . j}^{\prime n}+c p_{i . j+1}^{\prime n}+c p_{i . j-1}^{\prime n}+d=0 a
$$

where

$$
a=2\left(\frac{\Delta t}{(\Delta x)^{2}}+\frac{\Delta t}{(\Delta y)^{2}}\right), b=-\frac{\Delta t}{(\Delta x)^{2}}, c=-\frac{\Delta t}{(\Delta y)^{2}},
$$

$$
d=\frac{1}{\Delta x}\left[\left(u^{*}\right)_{i+1 / 2, j}^{n+1}-\left(u^{*}\right)_{i-1 / 2, j}^{n+1}\right]+\frac{1}{\Delta y}\left[\left(v^{*}\right)_{i, j+1 / 2}^{n+1}-\left(v^{*}\right)_{i, j-1 / 2}^{n+1}\right]
$$

In the above equations, the values of $\Delta x, \Delta y$ and $\Delta t$ are determine as below:

$$
\Delta x=L / n x-1, \Delta y=h / n y-1 \text { and } \Delta t=0.001 \mathrm{~s}
$$

where $n x$ the number of steps in $\operatorname{space}(x)$ and $n y$ the number of steps in $\operatorname{space}(y)$. The reason why $\Delta t=0.001 \mathrm{~s}$ is chosen illustrated in Fig. 4.

The SIMPLE algorithm has often been used to solve the problem of incompressible flow on a staggered grid. Its procedure is iterative, consisting of a guess and a corrector step. In the guess step, the velocity field is calculated from the momentum equations based on the estimated pressure field. In the corrector step, the pressure correction equation is derived and solved. Then, the velocity fields are corrected by using the variation in the pressure field. The velocity and pressure values are calculated iteratively to satisfy the continuity equation. The steps are stopped when convergence is achieved. More details about SIMPLE algorithm are shown below. The resulting numerical computation and simulation are solved by the SIMPLE algorithm using MATLAB programming.

1. Guess the pressure field $p^{*}$.

2. Solve the momentum equations to obtain $u^{*}$ and $v^{*}$.

3. Solve the pressure correction equation for $p^{\prime}$.

4. Calculate $p$ by adding $p^{\prime}$ to the previous value of $p^{*}$.

5. Calculate $u$ and $v$ from the velocity correction relations as follow.

$$
u=u^{*}+u^{\prime} \text { and } v=v^{*}+v^{\prime} .
$$

6. Solve the discretization for other scalar equations such as temperature, concentration etc., if they influence the flow field through fluid properties, source term etc. If they do not influence, it is better to calculate after the convergent solution is obtained.

7. Treat the corrected pressure $p$ as $p^{*}$ and return to step 2 and repeat the whole procedure until a convergent solution is achieve.

\subsection{Assignment of the Parameter Values}

The above algorithms are developed to solve the problem of the effect of gravitational acceleration on biomagnetic fluid flow and heat transfer in the channel under the influence in spatially varying magnetic field. The system of equations in (8) to (11) has been solved together with the appropriate boundary conditions. The dimensionless parameters are required to allocate the values for entering into this problem in order to continue to the derivation of the numerical results. For this problem, we assumed the fluid is the blood with $\rho^{*}=1050 \mathrm{~kg} / \mathrm{m}^{3}$ and $\mu^{*}=3.2 \times 10^{-3} \mathrm{kgm}^{-1} \mathrm{~s}^{-1}$ [14]. The fluid flows with the maximum velocity, $u_{r}^{*}=1.0 \mathrm{~ms}^{-1}$, the two parallel plates are located at a distance $h^{*}=1.0 \times 10^{-2} \mathrm{~m}$, and the Reynolds number, $R e$ is set to 200 . 
Table 1. Dimensionless parameter for magnetic number with $R e=200$

\begin{tabular}{ll}
\hline Magnetic Induction $\left(\boldsymbol{B}_{\boldsymbol{r}}^{*}\right.$, Tesla) & Magnetic Number \\
\hline 2 & 30.76 \\
4 & 61.52 \\
6 & 92.30 \\
8 & 123.0 \\
\hline
\end{tabular}

The present results are acquired for grid $31 \times 21$, i.e. 651 grid points. The magnetic field is located at the point $(a, b)=(2.5,-0.05)$. The points that we test are at $x=0$, $x=2.5, x=5.5$ and $x=10$. Tzirtzilakis [22] stated that there are two techniques to increase the magnetic number. By adding artifically created nanoparticles, the magnetization of blood will increase. So, the higher values of magnetic number $M n_{F}$ may rise. In this case, the blood has achieved magnetization of $M_{r}^{*}=60 \mathrm{~A} / \mathrm{m}$ [8]. On that account, the range of magnetic number, $M n_{F}$ is not only determined by magnetic induction but also by a magnetic field generated by a common magnetic dipole based on the physical problem.

A range is adopted for temperature field under the influence of magnetic field. The temperature of the upper plate is assumed as $T_{u}^{*}=43^{\circ} \mathrm{C}$ while the temperature for the lower plate is considered as $T_{l}^{*}=3.5^{\circ} C$ [4]. For these values of plate temperature, the temperature number $\varepsilon$ is equal to 8 . The Prandtl number is considered to be constant due to the viscosity $\mu^{*}$, the specific heat under a constant pressure $c_{p}^{*}$ and the thermal conductivity $k^{*}$ of any fluid are temperature dependent. Therefore, for the temperature range considered in this problem, it can be assumed that $\operatorname{Pr}=20$ when the measurements for fluid (blood) are $c_{p}^{*}=14.65 \mathrm{Jg}^{-1} \mathrm{~K}^{-1}$ and $k^{*}=2.2 \times 10^{-3} \mathrm{Jm}^{-1} \mathrm{~s}^{-1} \mathrm{~K}^{-1}$ and Eckert number, Ec is equal to $2.5057 \times 10^{-6}$. For effect of gravitational acceleration on biomagnetic fluid flow and heat transfer, we set Richardson number in the range $0 \leq R i \leq 3.55$ and the value of gravitational acceleration approximately $9.8 \mathrm{~ms}^{-2}$.

\section{Results and Discussion}

Fig. 3 illustrates the $u$ - velocity profile for various numbers of iterations, $K$ ranging from 4 to 300 . The $u$ velocity profile has converged to Couette flow solution at $K=300$.

Fig. 4 indicates the velocity profile for unsteady flow field at various times. These results are compared to the steady solution [8]. It is observes that the value of $\Delta t=0.001 \mathrm{~s}$ is acceptable for the present calculation. The calculation becomes unstable when $\Delta t$ is larger.

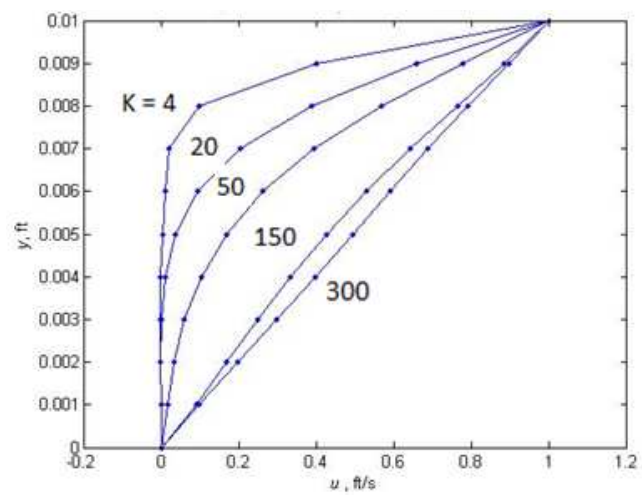

Figure 3. The $u$-velocity profile for various iteration numbers

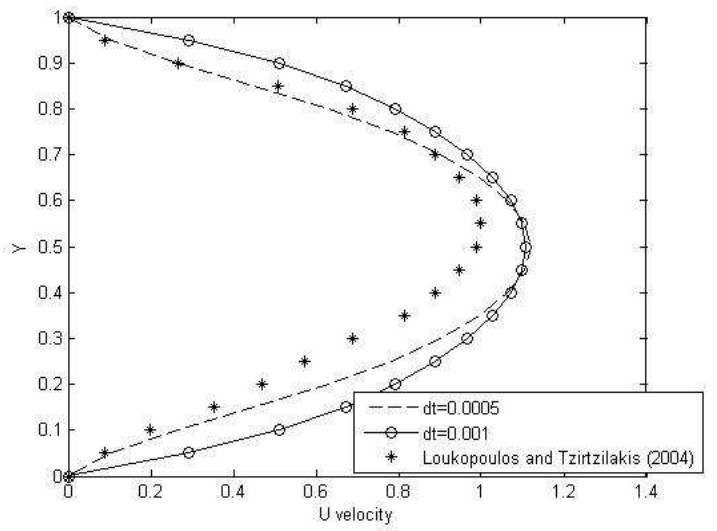

Figure 4. Velocity profile for unsteady flow field at various stages in time-stepping process

Fig. 5 depicts the axial velocity profile along specific locations in the channel for $B_{r}^{*}=8 T$. These results are compared with Loupopoulos and Tzirtzilakis [8]. At the point $x=0$, the axial velocity of the present method is equivalent to boundary condition used. At the point $x=2.5$, the present method gives approximately the same result as Loupopoulos and Tzirtzilakis [8]. But, at $x=5.5$, the flow pattern seems to contrast Loupopoulos and Tzirtzilakis [8]. Finally, the flow pattern of velocity at the outlet which is at the point $x=10$ matches nicely with Loupopoulos and Tzirtzilakis [8].

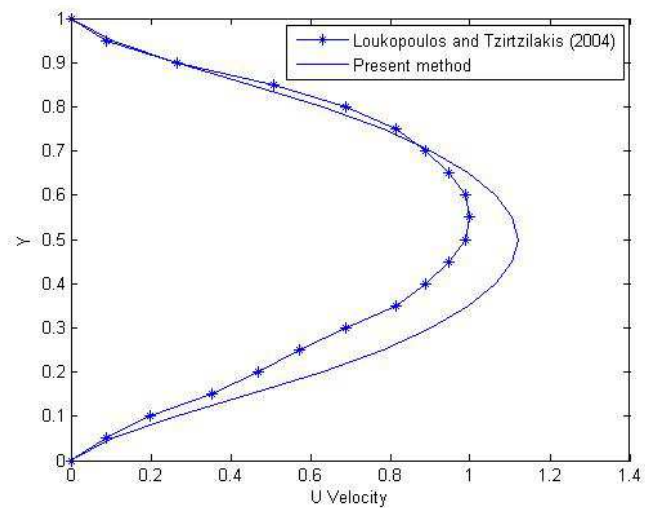

(a) $x=0$ 


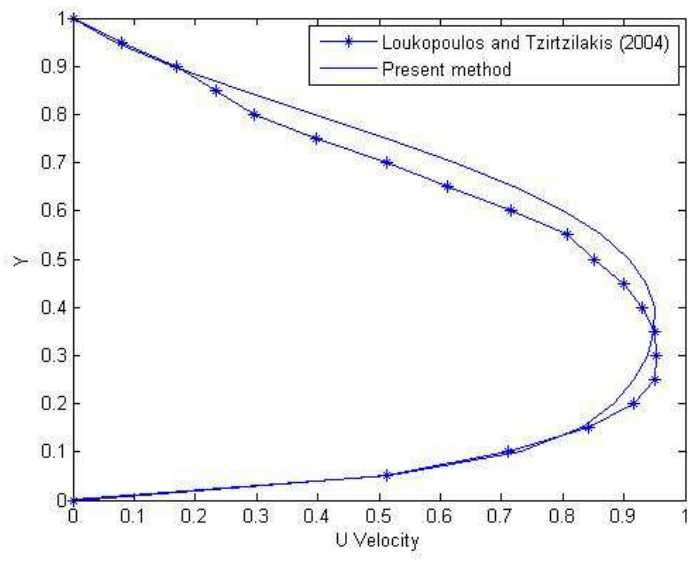

(b) $x=2.5$

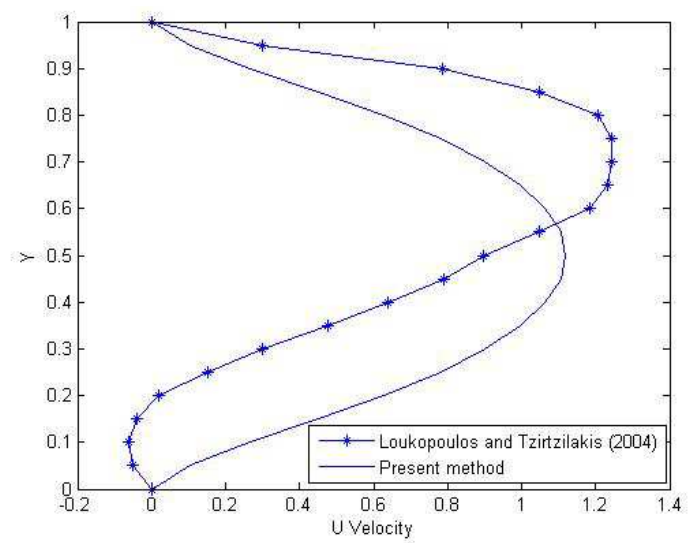

(c) $\quad x=5.5$

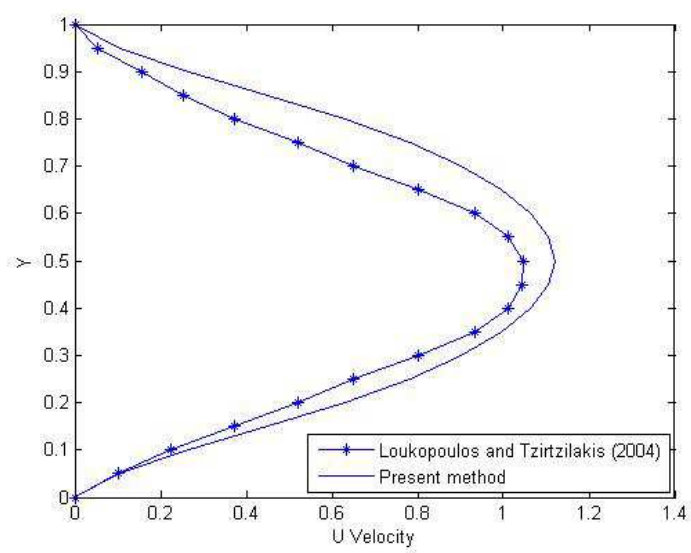

(d) $x=10$

Figure 5. The axial velocity profile along specific locations in the channel for $B_{r}^{*}=8 T$

Fig. 6 shows the effect of gravitational acceleration on the flow field in streamline velocity contour with and without magnetic field. From the figure, it can be seen that there is a distraction in the velocity field after a point of magnetic strength and the distraction becomes greater with the increase of magnetic field strength.

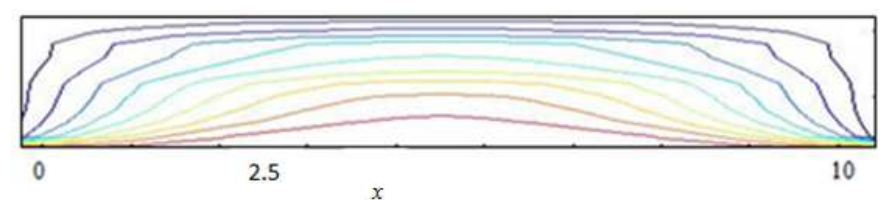

a)

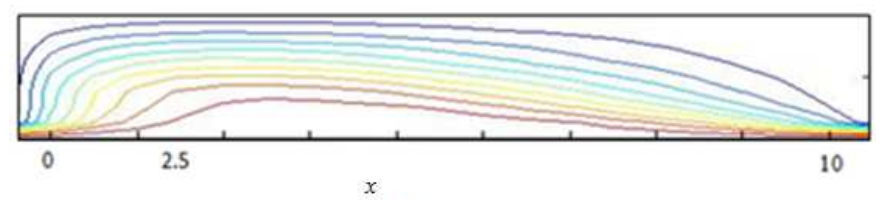

b) $2 T$

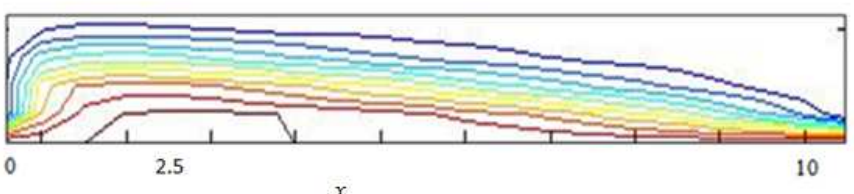

b) $4 T$

Figure 6. The effect of gravitational acceleration at $x=2.5$. (a) without magnetic field $b$ ) with magnetic field at $2 T$ and $4 T$

Fig. 7 indicates the $v$-velocity profile at $x=2.5$ with and without gravitational acceleration. For $v$ - velocity profile without gravitational acceleration, the velocity decreases from 0 to -1.34 and stays constant after $x=0.3$. With gravitational acceleration, the velocity decreases from 0 to -2.57 and then remains constant. These results show that gravitational acceleration will cause the velocity of fluid with magnetic field to drop more sharply. The graphs indicate some fluctuations at points $x=0.02$ to $x=0.15$. But the fluctuation reduces with the effect of gravitational acceleration.

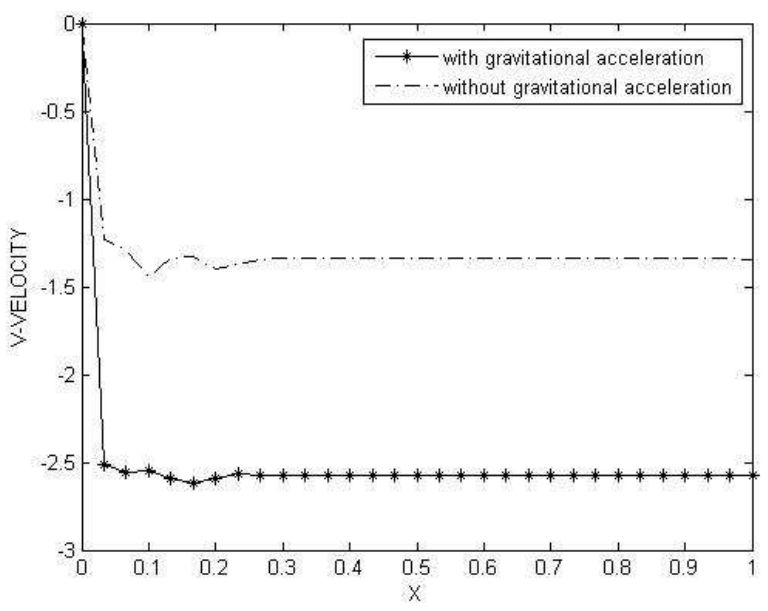

Figure 7. The effect of gravitational acceleration for the $v$-velocity profile at $x=2.5$ for $B_{r}^{*}=8 T$ 


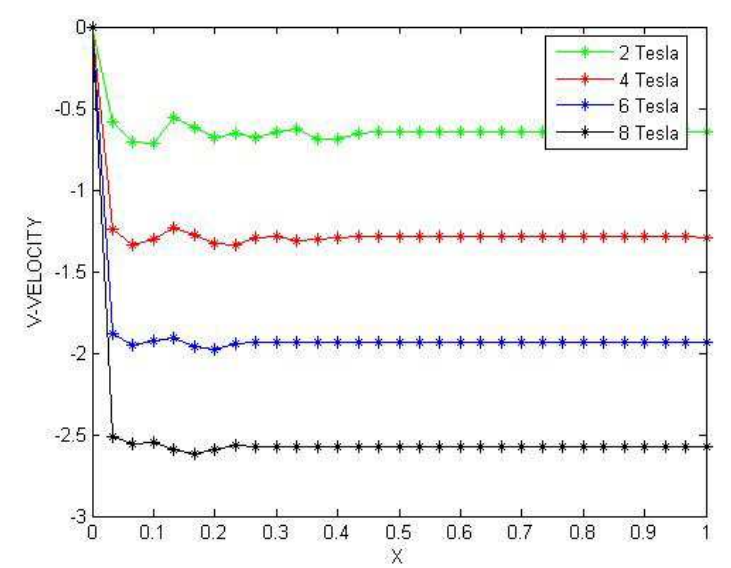

Figure 8. The effect of gravitational acceleration for the $v$ - velocity profile at $x=2.5$ for various magnetic field intensities

Fig. 8 shows the $v$-velocity profile at $x=2.5$ for various magnetic field intensities with $R i=3.55$. It is evident from the figure that the increase in magnetic field strength will cause the $v$ - velocity field to decrease drastically. The effect is very significant for magnetic field strength 8 Tesla where the $v$ - velocity decreases from 0 to -2.57 compared to the decrease from 0 to -0.64 at 2 Tesla.

Fig. 9 shows the $u$ - velocity profile with and without gravitational acceleration. From the figure, it can be seen that there is no change in the $u$-velocity profile.

The effect of gravitational acceleration for the axial velocity profile at $x=2.5$ for varying magnetic field strengths is illustrates in Fig. 10. For $B_{r}^{*}=2 T$ and $B_{r}^{*}=4 T$, the maximum axial velocity are about 0.77 . The axial velocity increases from 0.85 and 0.95 for $B_{r}^{*}=6 T$ and $B_{r}^{*}=8 T$ respectively. It shows that the velocity at the upper plate increases with the magnetic field strength.

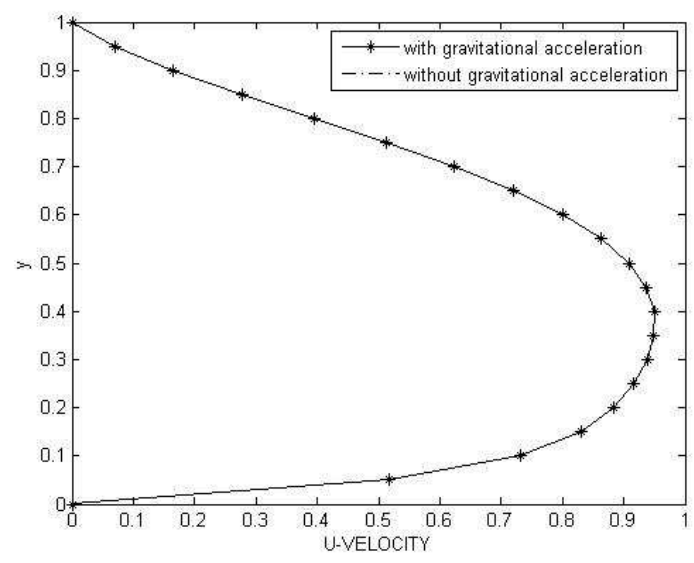

Figure 9. The effect of gravitational acceleration on the $u$-velocity profile at $x=2.5$ for $B_{r}^{*}=8 T$

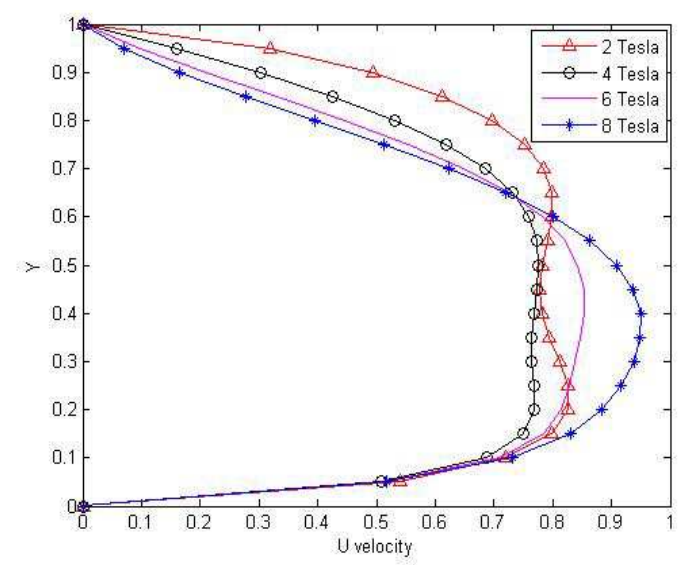

Figure 10. The effect of gravitational acceleration on the $u$-velocity profile at $x=2.5$ for various magnetic field intensities

Fig. 11 shows the temperature profiles at different parts of the channel. At the point $x=0$ which is at the inlet, the present method shows that the temperature result matches nicely as reported in Erwan [4]. At the point $x=2.5$, the temperature profile for our method increases but it remains a constant value from $y=0$ to $y=0.2$ about $5^{0} \mathrm{C}$ as in Erwan's study. At the points $x=3.1$ and $x=5.5$, the temperature profile retains a constant value from $y=0$ to $y=0.8$ at about $0^{\circ} \mathrm{C}$. In contrast Erwan's study shows the temperature increases at point $x=3.1$ and decreases at point $x=5.5$. The temperature disturbance occurs due to the addition of the magnetic field strength. The temperature disturbance shows slight deviations at the point $x=2.5$ between the inlet and outlet. But, the temperature disturbance is more at the points $x=3.1$ and $x=5.5$.

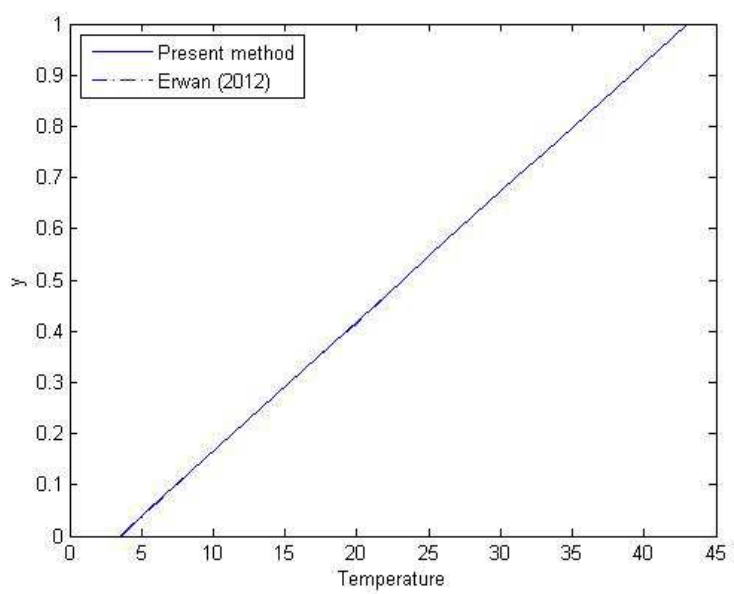

a) $\quad x=0$ 


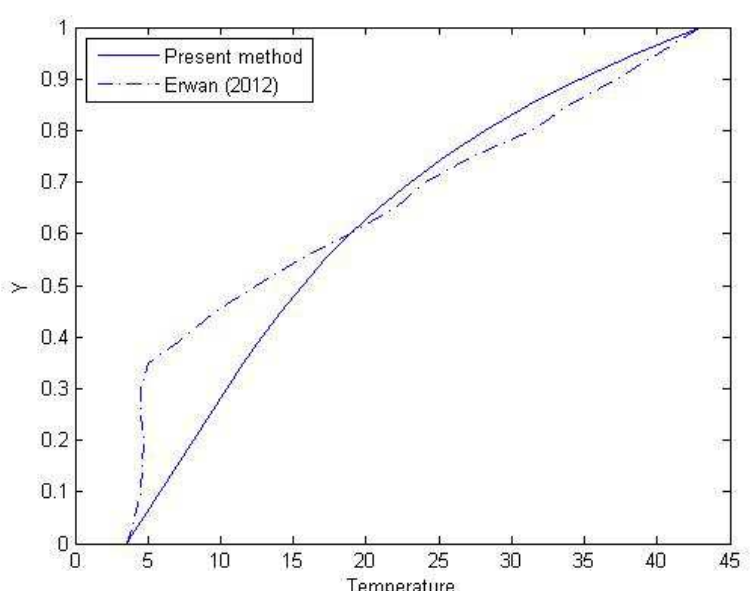

b) $x=2.5$

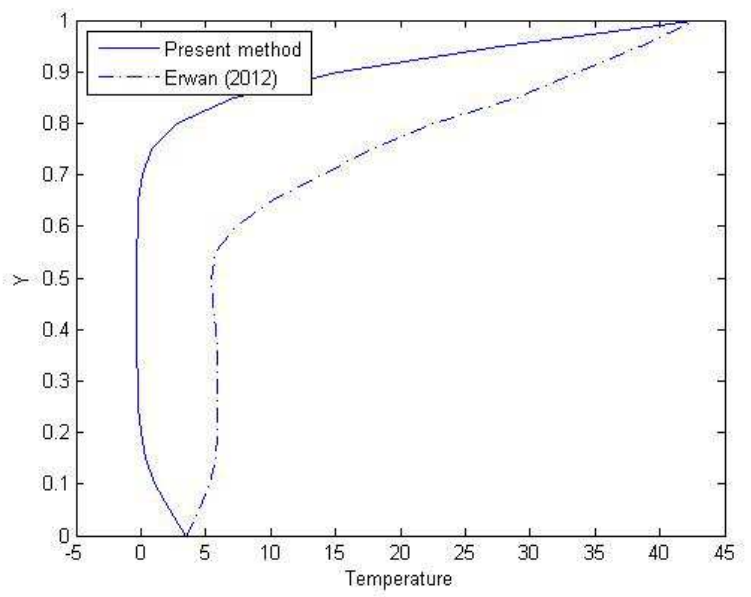

c) $x=3.1$

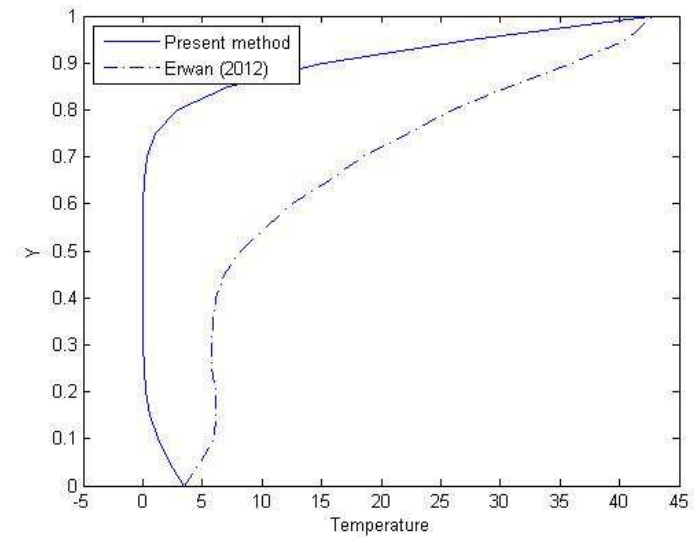

d) $\quad x=5.5$

Figure 11. The temperature profile along specific locations in the channel for $B_{r}^{*}=8 T$

The temperature profile at point $x=2.5$ for various magnetic field strengths is presented in Fig. 12. These results prove that the increment of magnetic field strength will cause an increase in the temperature profile.



Figure 12. The temperature profile at $x=2.5$ for various magnetic field intensities

Fig. 13 illustrates the effect of gravitational acceleration for the temperature profile at $x=2.5$ against that without gravitational acceleration. The temperature field remains constant from $y=0$ to $y=0.95$ at about $7.94^{\circ} \mathrm{C}$. This result shows that gravitational acceleration causes big influence to the temperature profile.

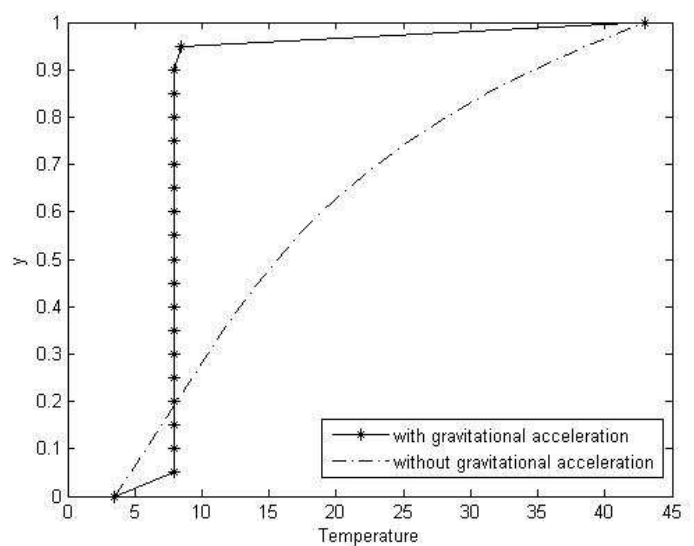

Figure 13. The effect of gravitational acceleration for the temperature profile at $x=2.5$ for $B_{r}^{*}=8 T$

The temperature distribution shows that a little difference to the changing for various magnetic field strengths in Fig. 14.

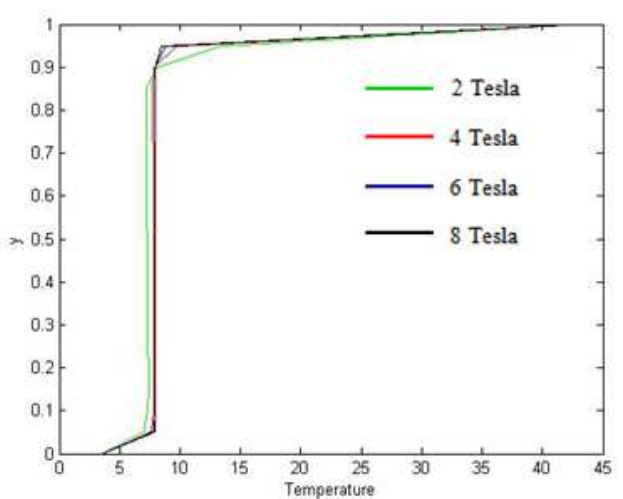

Figure 14. The effect of gravitational acceleration for the temperature profile for various magnetic field intensities 


\section{Conclusion}

In this study numerical solutions of biomagnetic fluid flow under the influence of varying magnetic field are presented. The flow considered is unsteady, non-isothermal and based on the principle of FHD. This model is solved using finite difference numerical technique based on SIMPLE algorithm on the staggered grid system. The main objective of this paper is to employ the model of biomagnetic fluid using the principle of ferrohydrodynamics to investigate the effect of gravitational acceleration on unsteady fluid flow. From the results, it can be concluded that the magnetic field and gravitational acceleration influence the fluid flow behaviour. The increment of magnetic field strength causes the velocity and temperature fields to increase. A vortex appears near the lower plate below where the magnetic source is located. Besides, it also shows that the drastic changes in the $v$ velocity field as a result of gravitational acceleration. The velocity decreases and remains constant after a certain point.

\section{Acknowledgements}

Financial supports provided by Vot 01G31, Research University Grant Scheme, Universiti Teknologi Malaysia, are gratefully acknowledged.

\section{References}

[1] Anderson, Computational Fluid Dynamics: The Basic with Applications, McGraw-Hill, Inc., 1995.

[2] Andersson, H. I. and Valnes, O. A., "Flow of a heated ferrofluid over a stretching sheet in the presence of a magnetic dipole", Acta Mech., 128(39), 1998.

[3] Bashtovoy, V. G., Berkovsky, B. M. and Vislovich, A. N., "Introductionto Thermomechanics of Magnetic Fluids", Hemisphere, Springer-Verlag, Berlin, 1988.

[4] Erwan Hafizi Kasiman, "Mixed formulation for Navier-Stokes equations with magnetic effect in rectangular channel", Masters Thesis. Department of Mathematical Sciences, Universiti Teknologi Malaysia, Johor Bahru, 2012.

[5] Fertman, V. E., Magnetic Fluids Guidebook: Properties and Applications, Hemisphere, New York, 1990.

[6] Higashi, T., Yamagishi, A., Takeuchi, T., Kawaguchi, N., Sagawa, S., Onishi, S and Date, M., "Orientation of erythrocytes in a strong static magnetic field", J. Blood, 82(1328), 1993.

[7] Haik, Y., Chen, J. C. and Pai, V. M., Development of bio-magnetic fluid dynamics, Proceedings of the $I X$ International Symposium on Transport Properties in Thermal Fluids Engineering, Singapore, Pacific Center of Thermal Fluid Engineering, S. H. Winoto, Y. T. Chew, N. E. Wijeysundera, eds, Hawaii, U.S.A., June 25-28, 1996, pp 121-126.

[8] Loukopoulos, L. C. and Tzirtzilakis, E. E., "Biomagnetic channel flow in spatially varying magnetic field", Int. J. Eng.
Sci., 42, 2004, pp $571-590$.

[9] Muthucumaraswamy, R., Raj, M. S. and Subramanian, V. S. A., "Heat transfer effects on accelerated vertical plate with variable temperature and mass flux", ACTA TECHNICA CORVINIENSIS, 2010.

[10] Nursalasawati Rusli, "The computational modeling and simulation of two and three dimensional models of biomagnetic fluid flow in an artery", Ph.D Thesis, Department of Mathematical Sciences, Universiti Teknologi Malaysia, Johor Bahru, 2012.

[11] Papadopoulos, P. K and Tzirtzilakis, E. E., "Biomagnetic flow in a curved square duct under the influence of an applied magnetic field”, Phys. Fluids, 16(8), 2004, pp 2952 - 2962.

[12] Stemme, O., "Magnetic wound treatment", in Magnetism in Medicine, edited by W. Andra and H. Nowak, Wiley, Berlin, 1998, pp 489-494.

[13] Saha, S., Saha, G., Ali, M. and Islam, M. Q., "Combined free and forced convection inside a two-dimensional multiple ventilated rectangular enclosure", ARPN Journal of Engineering and App. Sciences, 1(3), 2006.

[14] Tzirtzilakis, E. E, "A simple numerical methodology for BFD problems using stream function vorticity formulation", Communications in Numer. Methods in Engineering, 2000, pp $1-6$.

[15] Tzirtzilakis, E. E., Kafoussias, N. G. and Hatzikonstantinou, P. M., "Biomagnetic fluid flow in a rectangular duct", 4th GRACM Congress on Computational Mechanics, Patras-Greece, 27-29 June, 2002.

[16] Tzirtzilakis, E. E. and Kafoussias, N. G., "Biomagnetic fluid flow over a stretching sheet with nonlinear temperature dependent magnetization", Z. angew. Math. Phys., 54, 2003, pp $551-565$.

[17] Tzirtzilakis, E. E. and Tanoudis, G. B., "Numerical study of biomagnetic fluid flow over a stretching sheet with heat transfer", Int. J. Numer. Methods Heat Fluid Flow, 13(830), 2003.

[18] Tzirtzilakis, E. E., Sakalis, V. D., Kafoussias, N. G. and Hatzikonstantinou, P. M., "Biomagnetic fluid flow in a 3D rectangular duct", Int. J. Numer. Meth. Fluids, 44, 2004, pp 1279-1298.

[19] Tzirtzilakis, E. E., "A mathematical model for blood flow in magnetic field", Phys. Fluids, 17(7), 2005.

[20] Tzirtzilakis, E. E. and Loukopoulos, V. C., "Biofluid flow in a channel under the action of a uniform localized magnetic field", Comput. Mech., 36(5), 2005, pp 360 - 374.

[21] Tzirtzilakis, E. E., Xenos, M., Loukopoulos, V. C. and Kafoussias, N. G., "Turbulent biomagnetic fluid flow in a rectangular channel under the action of a localized magnetic field”, Int. Journal of Engineering Science, 44, 2006, pp 1205 1224.

[22] Tzirtzilakis, E. E., "Biomagnetic fluid flow in a channel with stenosis”, Physica D., 237(1), 2007, pp 66 - 81.

[23] Tzirtzilakis, E. E. and Xenos, M. A., "Biomagnetic fluid flow in a driven cavity", Meccanica, 48, 2013, pp $187-200$. 